\title{
FIRST REPORT OF ACANTHAMOEBA GENOTYPE T8 HUMAN KERATITIS
}

\author{
ERIKA Orosz ${ }^{1 *}$, Nóra SZENTMÁrY ${ }^{2,3}$, HubA J. KISS ${ }^{2}$, ÁGNES FARKAS $^{4}$, \\ ISTVÁN KUCSERA ${ }^{1}$ and ZOLTÁN ZSOLT NAGY ${ }^{2}$ \\ ${ }^{1}$ Department of Parasitology, National Public Health Institute, Budapest, Hungary \\ ${ }^{2}$ Department of Ophthalmology, Semmelweis University, Budapest, Hungary \\ ${ }^{3}$ Department of Ophthalmology, Saarland University Medical Center, \\ Homburg/Saar, Germany \\ ${ }^{4}$ Division of Virology, National Public Health Institute, Budapest, Hungary
}

(Received: 23 June 2017; accepted: 15 November 2017)

\begin{abstract}
Acanthamoeba has a worldwide distribution in the environment and it is capable of causing a painful sight-threatening disease of the cornea designated as Acanthamoeba keratitis (AK). Nowadays, the cases of AK have surged all over the world along with its disease burden due to increasing use of contact lenses used not only for optical correction but also for cosmetic purposes. In our present work, epithelial abrasion of a 27-year-old female soft contact lens wearer with keratitis was examined. Genotype identification was carried out with a real-time fluorescence resonance energy transfer polymerase chain reaction (PCR) assay based on sequence analysis of the 18S rRNA gene. Genotyping allowed the identification of a T8 group isolate. The analysis confirmed the importance of a complete diagnostic protocol, including a PCR assay, for the clinical diagnosis of AK from human samples. Acanthamoeba T8 should be considered as potential causative organism in keratitis in human.
\end{abstract}

Keywords: $18 \mathrm{~S}$ rRNA, Acanthamoeba, real-time PCR, hybridization probes, fluorescence resonance energy transfer PCR, sequence analysis

\section{Introduction}

Acanthamoeba has a worldwide distribution in the environment [1-4]. Acanthamoeba keratitis (AK) infections occur after water exposure or a history of swimming in lakes, following contact with soil or plants, or due to contact lens wear for optical or cosmetic purposes [5-7]. Based on rRNA gene sequences, the genus Acanthamoeba is divided into 20 different genotypes to date (T1-T20). The first description of amoebic keratitis (AK) and granulomatous amebic encephalitis (GAE) in 1975 was performed by Jones et al. [8].

*Corresponding author; E-mail: orosz.erika@oki.antsz.hu 
Certain species of the genus Acanthamoeba can cause amoebic keratitis in healthy humans [9]; in immunocompromised individuals, it can cause the fatal GAE [10].

In the past few years, from ecological diagnostic and clinical therapy viewpoints, a demand raised for a polymerase chain reaction (PCR) method, which can be used for Acanthamoeba spp. studies [11]. Real-time PCR allows specific detection of the amplification by binding with fluorescence-labeled probes, and further downstream analysis is not required. This considerably reduces the time required for detection. In addition, the closed reaction tube minimizes the chance of cross-contamination and the assay output is quantitative and qualitative [12].

Our aim was to present genotypic characterization of an Acanthamoeba spp. isolated from human corneal epithelium and contact lens storage case of a patient with clinical signs of Acanthamoeba keratitis.

\section{Materials and Methods}

\section{Isolation}

Acanthamoeba was isolated from human corneal epithelium (following focal corneal abrasion) of a patient with clinical signs of AK (multifocal stromal infiltrates and ring infiltrate) and from contact lens storage case of the same patient.

\section{Culture-confirmed detection method}

The corneal epithelial sample and fluid from contact lens storage case were then transferred to Page's agar plates overlaid with heat-killed Escherichia coli and cultured at $37^{\circ} \mathrm{C}$ for 10 days. The morphology of trophozoites and cysts were studied by light microscopy, according to Page [13]. Plates were monitored for growth of amoeba microscopically, from 72 to $96 \mathrm{~h}$ for the presence of Acanthamoeba spp. cysts and trophozoites under $320 \times$ and $400 \times$ magnification.

\section{Molecular methods}

The Acanthamoeba species were isolated by dilution method. For this purpose, the samples of corneal scrapings were suspended in $400 \mu \mathrm{l}$ physiological saline solution $(0.85 \%)$. After preparation, the DNA extraction was treated with High Pure PCR Template Preparation Kit (Germany), according to the instructions 
of the manufacturer. The DNA amplification was performed using genus-specific primers and genus-specific-fluorescence resonance energy transfer hybridization probes, previously described by Orosz et al. [14]. Each experiment included one reaction mixture without DNA as a negative control; positive control and each specimen were run in duplicate for real-time PCR assay in parallel.

PCR products were purified with PCR Clean up-M Kit (Viogene, Sunville, CA). The sequence of each amplicon was determined by cycle sequencing with primers for the $5^{\prime}$-NTR region and with primers with BigDye Terminator v3.1 Cycle Sequencing Kit (Applied Biosystems, Germany), according to the manufacturer's instruction. The electrophoresis was carried out on Applied Biosystems 3500 Genetic Analyzer.

The 5'-NTR and VP1 gene sequences were subjected to nucleotidenucleotide BLAST analysis [15] using the online server at the National Center for Biotechnology Information (http://blast.ncbi.nlm.nih.gov/Blast).

The unknown sequences were aligned with known published sequences of the major genotypes using the alignment program MULTALIN (http://multalin. toulouse.inra.fr/multalin) [16]. The genotypes of samples were determined based on this comparison.

The phylogenetic tree was constructed by the neighbor-joining method of genetic distance calculated by the MEGA 5 (http://www.megasoftware.net) [17].

\section{Results}

\section{Cultivation}

Microscopically, the cultivation was successful for both samples. Both investigated samples revealed Acanthamoeba that was able to grow at $36{ }^{\circ} \mathrm{C}$, which is the approximate temperature of the human host (Figure 1). Further examination of the obtained results was carried out by FRET PCR.

\section{Molecular analysis}

PCR amplification from both corneal epithelium and fluid from contact lens storage was succesfull. Thereafter, the Acanthamoeba positive samples, detected by PCR method, were sequenced to identify the species. Sequence analysis using a BLAST search indicated an identity of $>98 \%$ with Acanthamoeba $18 \mathrm{r}$ rRNA gene reference sequences. All obtained sequences of amoeba isolates from the case belonged to the T8 genotype Acanthamoeba spp. (NCBI Acanthamoeba healy MF065931.1). Neighbor-joining analysis inferred relationships between the PCR 

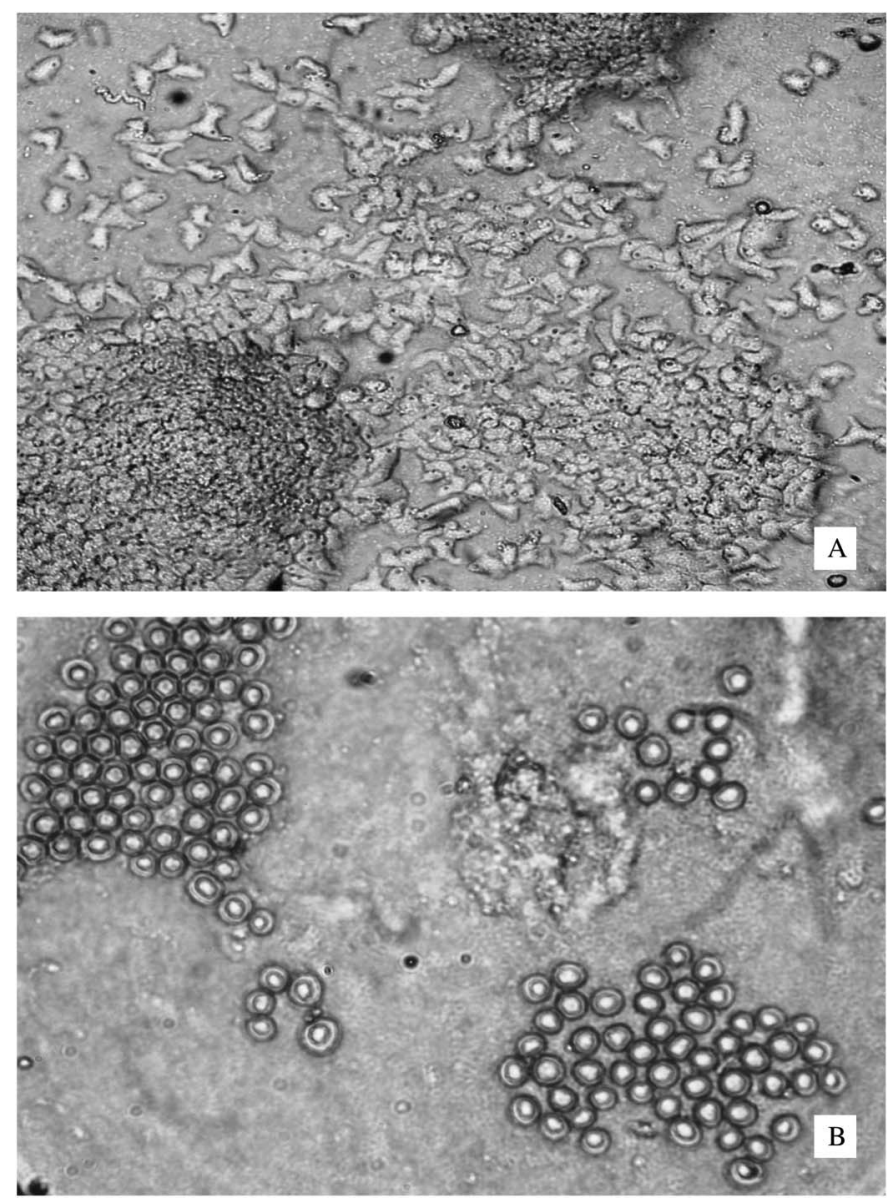

Figure 1. Photomicrograph of Acanthamoeba trophozoites and cysts with $320 \times(\mathrm{A})$ and $400 \times(\mathrm{B})$ magnification. Photographer: Erika Orosz

products isolated from corneal scrapings and reference strains obtained from NCBI GenBank, shown in Figure 2, respectively.

\section{Discussion and conclusions}

Acanthamoeba identification can be rapidly achieved using real-time molecular methods. For diagnostic purposes, the detection of Acanthamoeba at the genus level is sufficient to recognize whether an individual is infected $[18,19]$. 


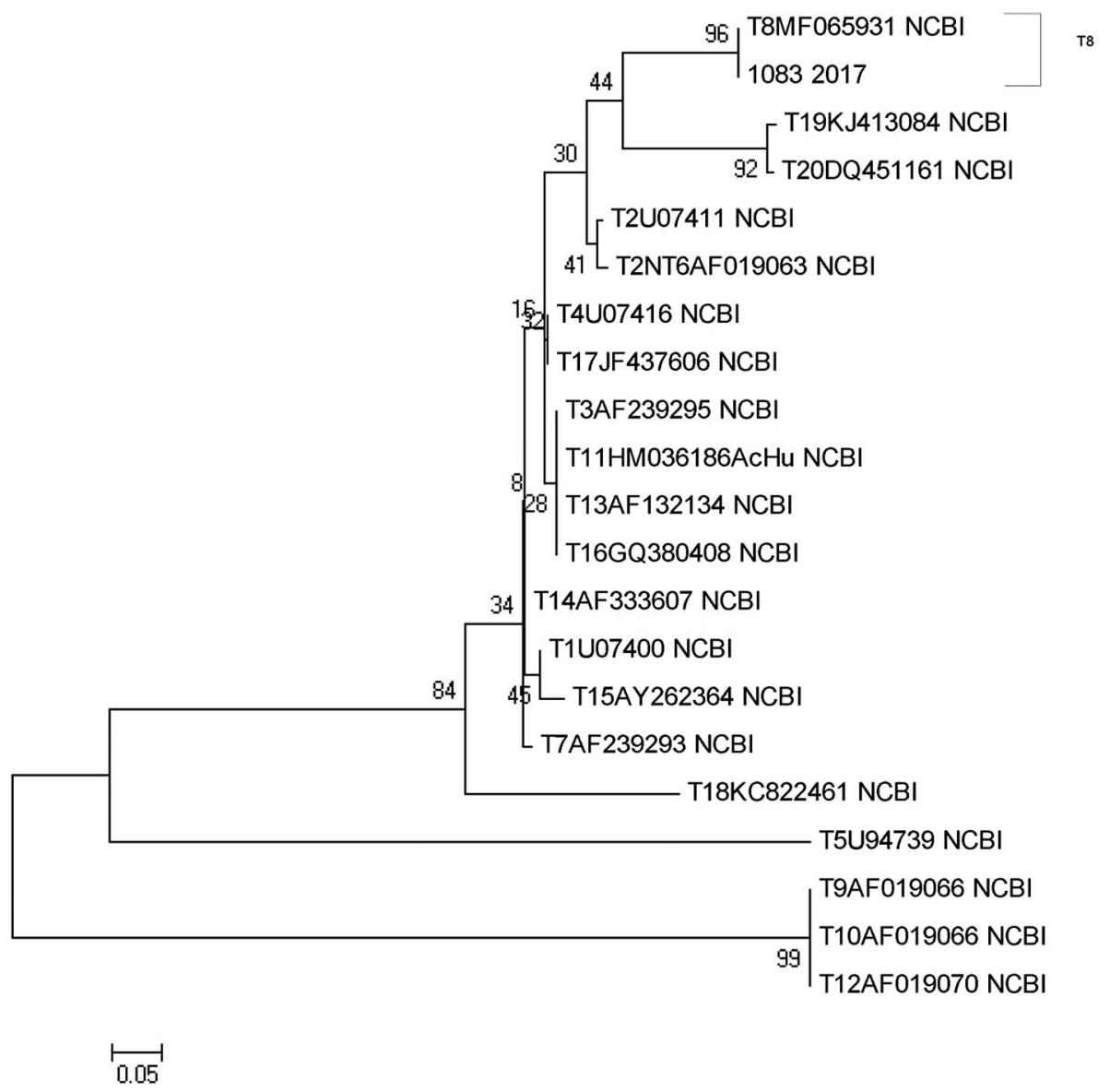

Figure 2. Phylogenetic relations of Acanthamoeba species PCR product 1083_2017 and reference strains from NCBI GenBank inferred by neighbor-joining analysis from pairwise comparisons (180-bp fragments)

A more precise differentiation of genotype is beneficial for better understanding of Acanthamoeba taxonomy and also facilitates the correct identification.

These organisms have gained attention from the broad scientific community studying environmental biology, molecular biology, and biochemistry. The culture-confirmed detection method is slow $(96 \mathrm{~h})$ and can detect Acanthamoeba only at genus level, whereas the molecular genotype analysis is faster (real-time PCR $4 \mathrm{~h}$ ). Based on rRNA gene sequences, the genus Acanthamoeba is divided into 20 different genotypes to date (T1-T20). Each genotype exhibits $5 \%$ or more sequence divergence [9, 20, 21]. 
In conclusion, in this study, we reveal the genetic diversity of Acanthamoeba spp. existing in keratitis patients using the nuclear small subunit ribosomal RNA (18S rRNA). To the best of our knowledge, this is the first report on T8 genotype Acanthamoeba spp., causing AK in human. The presence of Acanthamoeba T8 should also be considered as potential causative organism of contact lens-associated keratitis.

\section{Conflict of Interest}

There is no conflict of interest. EO assures that there are no links with a company whose product is mentioned in the article or a company that distributes a competing product. The presentation of the topic is independent and the presentation of the content is product-neutral.

\section{References}

1. Podlipaeva, J. I., Shmakova, L. A., Gilichinski, D. A., Goodkov, A. V.: Heat shock protein of hsp70 family revealed in some contemporary freshwater amoebids and in Acanthamoeba sp. from cysts isolated from permafrost samples. Tsitologiya 48, 691-694 (2006).

2. Shatilovich, A. V, Shmakova, L. A., Gubin, S. V, Goodkov, A. V., Gilichinsky, D. A.: Viable protozoa in late Pleistocene and Holocene permafrost sediments. Doklady Biol Sci 401, 136-138 (2005).

3. Nuprasert, W., Putaporntip, C., Pariyakanok, L., Jongwutiwes, S.: Identification of a novel T17 genotype of Acanthamoeba from environmental isolates and T10 genotype causing keratitis in Thailand. J Clin Microbiol 48, 4636-4640 (2010).

4. Stothard, D. R., Schroeder-Diedrich, J. M., Awwad, M. H., Gast, R. J., Ledee, D. R., Rodriguez-Zaragoza, S., Dean, C. L., Fuerst, P. A., Byers, T. J.: The evolutionary history of the genus Acanthamoeba and the identification of eight new 18S rRNA gene sequence types. J Eukaryot Microbiol 45, 45-54 (1998).

5. Kilvington, S., White, D. G.: Acanthamoeba: Biology, ecology and human disease. Rev Med Microbiol 5, 12-20 (1994).

6. Walochnik, J., Aichelburg, A., Assadian, O., Steuer, A., Visvesvara, G., Vetter, N.: Granulomatous amoebic encephalitis caused by Acanthamoeba amoebae of genotype $\mathrm{T} 2$ in a human immunodeficiency virus negative patient. J Clin Microbiol 46, 338-340 (2008).

7. Sharifi, N., Botero-Kleiven, S., Ohman, D., Barragan, A., Winiecka-Krusnell, J.: Genotypic characterization of Acanthamoeba spp. causing ocular infections in Swedish patients: Identification of the T15 genotype in a case of protracted keratitis. Scand J Infect Dis $\mathbf{4 2}$, 781-786 (2010).

8. Jones, D. B., Visvesvara, G. S., Robinson, N. M.: Acanthamoeba polyphaga keratitis and Acanthamoeba uveitis associated with fatal meningoencephalitis. Trans Ophthalmol Soc UK 95, 221-231 (1975). 
9. Rivera, W. L., Adao, D. E. V.: 18S ribosomal DNA genotypes of Acanthamoeba species isolated from contact lens cases in the Philippines. Parasitol Res 105, 1119-1124 (2009).

10. Azzam, R., Badenoch, P. R., Francis, M. J., Fernandez, C., Adamson, P. J., Dendle, C., Woolley, I., Robson, J., Korman, T. M., Graham, M.: Acanthamoeba encephalitis: Isolation of genotype T1 in mycobacterial liquid culture medium. J Clin Microbiol, 53, 735-739 (2015).

11. Corsaro, D., Walochnik, J., Köhsler, M., Rott, M. B.: Acanthamoeba misidentification and multiple labels: Redefining genotypes T16, T19, and T20 and proposal for Acanthamoeba micheli sp. nov. (genotype T19). Parasitol Res 114, 2481-2490 (2015).

12. Orosz, E., Farkas, Á., Kucsera, I.: Laboratory diagnosis of Acanthamoeba keratitis in Hungary. Acta Microbiol Immunol Hung 63, 293-299 (2016).

13. Page, F. C.: A New Key to Freshwater and Soil Gymnamoebae. Freshwater Biological Association, Cumbria, UK, 1988, p. 122.

14. Orosz, E., Farkas, Á., Ködöböcz, L., Becságh, P., Danka, J., Kucsera, I., Füleky, G.: Isolation of Acanthamoeba from the rhizosphere of maize and lucerne plants. Acta Microbiol Immunol Hung 60, 29-39 (2013).

15. Altschul, S. F., Gish, W., Miller, W., Myers, E. W., Lipman, D. J.: Basic local alignment search tool. J Mol Biol 215, 403-410 (1990).

16. Corpet, F.: Multiple sequence alignment with hierarchical clustering. Nucleic Acids Res 16, 10881-10890 (1988).

17. Tamura, K., Peterson, D., Peterson, N., Stecher, G., Nei, M., Kumar, S.: MEGA5: Molecular evolutionary genetics analysis using maximum likelihood, evolutionary distance, and maximum parsimony methods. Mol Biol Evol 28, 2731-2739 (2011).

18. Grün, A. L., Stemplewitz, B., Scheid, P.: First report of an Acanthamoeba genotype T13 isolate as etiological agent of a keratitis in humans. Parasitol Res 113, 2395-2400 (2014).

19. Hajialilo, E., Behnia, M., Tarighi, F., Niyyati, M., Rezaeian, M.: Isolation and genotyping of Acanthamoeba strains (T4, T9, and T11) from amoebic keratitis patients in Iran. Parasitol Res 115, 3147-3151 (2016).

20. Alves, D. S., Moraes, A. S., Nitz, N., de Oliveira, M. G., Hecht, M. M., Gurgel-Gonçalves, R., Cuba, C. A.: Occurrence and characterization of Acanthamoeba similar to genotypes T4, T5, and T2/T6 isolated from environmental sources in Brasília. Exp Parasitol 131, 239-244 (2012).

21. Siddiqui, R., Khan, N. A.: Biology and pathogenesis of Acanthamoeba. Parasite Vector 5, 6 (2012). 